\title{
Treatment-induced amenorrhoea for patients with HER2-positive early breast cancer: does it matter?
}

\author{
Giuseppe Gullo, Jack P. Gleeson \\ Department of Medical Oncology, St. Vincent's University Hospital, Elm Park, Dublin 4, Ireland \\ Correspondence to: Jack P. Gleeson. Department of Medical Oncology, St. Vincent's University Hospital, Elm Park, Dublin 4, Ireland. \\ Email: jack.gleeson@hotmail.com. \\ Comment on: Lambertini M, Campbell C, Bines J, et al. Adjuvant Anti-HER2 Therapy, Treatment-Related Amenorrhea, and Survival in \\ Premenopausal HER2-Positive Early Breast Cancer Patients. J Natl Cancer Inst 2018. [Epub ahead of print].
}

Submitted Sep 05, 2018. Accepted for publication Sep 11, 2018.

doi: $10.21037 /$ tcr.2018.09.06

View this article at: http://dx.doi.org/10.21037/tcr.2018.09.06

The long-term outcome of patients with HER2-positive (HER2+) early breast cancer (EBC) has improved dramatically since the introduction of HER2-targeted therapies as part of the systemic standard of care (1-4). Consequently, survivorship issues, such as treatment related amenorrhoea (TRA) for younger patients, are increasingly important considerations for their impact on quality of life (QoL), however the prognostic implications of TRA remain unknown. Current standard of care for these patients involves a combination of chemotherapy and trastuzumab since the publication of the pivotal BCIRG 006 study, the HERA trial and the intergroup analysis of NCCTG N9831 and NSABP B-31, while the addition of other HER2-targeted agents, especially the monoclonal antibody pertuzumab, is still debated (3-6).

The correlation between TRA and HER2-targeted agents, including the monoclonal antibodies trastuzumab and pertuzumab and the small molecule lapatinib, as well as their impact on clinical outcomes, remain undefined. In theory, a period of TRA might lead to the withdrawal of hormone-induced stimulation of breast cancer cells, a cessation of cell growth and ultimately tumour-cell apoptosis and death, with resulting improvements in patient outcomes. The biological and clinical impact of TRA is expected to be higher on tumours that express hormone receptors, i.e., oestrogen and/or progesterone receptors positive $(\mathrm{HR}+)$ in addition to being classified as HER2positive. Whether TRA has any effect on the downstream activities of HER2-associated intracellular molecular pathways is unknown. Literature on this topic and directed studies are lacking, and in their paper Lambertini and coll. attempt to assess the prognostic effect of TRA in a retrospective unplanned analysis of prospectively collected data from the Adjuvant Lapatinib and/or Trastuzumab Treatment Optimization (ALTTO) study $(6,7)$.

ALTTO was an open-label phase III randomised trial in patients with HER2+ EBC in the adjuvant setting (6). Patients were randomly assigned $(1: 1: 1: 1)$ to four treatment groups, each of 1-year duration; trastuzumab (T) alone, lapatinib $(\mathrm{L})$ alone, sequential use of the 2 agents $(\mathrm{T} \rightarrow \mathrm{L})$ or both agents in combination $(\mathrm{T}+\mathrm{L})$. The $\mathrm{L}$ alone arm was closed in 2011 at the first interim analysis due to safety concerns and a lack of efficacy. The patients involved were those with centrally confirmed HER2+ non-metastatic invasive breast cancer, with either nodepositive disease or tumour size $\geq 1 \mathrm{~cm}$. 8,381 patients were enrolled and over 2,000 patients randomised to each arm in the study. In their study, Lambertini and coll. analysed the prospectively collected data on TRA, defined as those who at the 37-week visit did not meet the criteria for premenopausal status (less than 6 months since the last menstrual period, no prior bilateral ovariectomy, and not on an oestrogen-replacement, or as biochemical evidence of premenopausal status as per local guidelines) having previously met the same criteria at the time they were randomised in the study (7). Disease-free survival (DFS) was the primary outcome measure of the ALTTO study, with overall survival (OS) as a secondary endpoint.

The aim of the analysis conducted by Lambertini and coll. was to describe TRA rates after chemotherapy combined with trastuzumab and/or lapatinib, and to evaluate the prognostic effect in terms of DFS and OS of its 
occurrence in premenopausal women with $\mathrm{HR}+$ and HRHER2 + EBC. Given the lack of data and literature in this area, and the sound rationale for this analysis, the Authors of this study must be commended for their effort to assess and report on TRA rates and their prognostic implications. However, as ALTTO failed to meet its primary endpoint of DFS and one of the arms was even closed at the first interim analysis, among other limitations of the analysis by Lambertini and coll., it is difficult to draw definitive conclusions and while there are interesting and important points raised and questions posed by the Authors' findings, the results described here should be taken with caution.

The rationale for relating TRA to the HER2-targeted agents used in the study ( $\mathrm{T} \& \mathrm{~L}$ ) appears to be tenuous, given their mechanisms of action, the role of the HER family of proteins and the downstream pathways excessively activated by HER2 overexpression. The Authors suggest that the high rates of TRA seen across the four study arms $(2,047 / 2,844=72.0 \%)$ would indicate that these agents played a role in TRA development. There was a small absolute difference between the TRA rates across the four study arms; $\mathrm{T}+\mathrm{L}$ arm $(523 / 706=74.1 \%), \mathrm{T}$ alone $\operatorname{arm}(496 / 703=70.6 \%)$, the $\mathrm{L}$ alone $\operatorname{arm}(505 / 691=73.1 \%)$ and the $\mathrm{T} \rightarrow \mathrm{L}$ arm $(523 / 744=70.3 \%)$. Dual agent use should result in some difference in TRA if there is a true causative link, and here we see that the highest rate of TRA (74.1\%) is indeed seen in the $\mathrm{T}+\mathrm{L}$ arm. One could further argue that lapatinib is more likely to cause TRA than trastuzumab (rates $73.1 \%$ vs. $70.6 \%$, respectively), given the $+2.5 \%$ absolute difference between these two arms, and that at the 37-week reassessment point in the $\mathrm{T} \rightarrow \mathrm{L}$ arm (TRA rate: $70.3 \%$ ), the impact of lapatinib on TRA had not yet been seen. However, despite a total of 2,844 patients, equivalently spread across the four study arms, involved in the landmark analysis, the analysis did not find a statistically significant difference of developing TRA $(\mathrm{P}=0.64)$ and the differences observed among arms remain purely numerical. The factors which did confer a statistically significant risk of developing TRA in the multivariate analysis were those entirely unrelated to HER2-targeted agents, such as older age at diagnosis (adjusted OR: 2.84, 95\% CI: $1.93-4.17, \mathrm{P}<0.001$ ), addition of taxanes to anthracyclinebased chemotherapy (adjusted OR: 1.92, 95\% CI: 1.44-2.56, $\mathrm{P}<0.001$ ), administration of docetaxel, carboplatin, and trastuzumab (TCH) regimen (adjusted OR: 2.24, 95\% CI: $1.18-4.27, \mathrm{P}=0.01$ ), and use of adjuvant endocrine therapy (adjusted OR: 2.84, 95\% CI: 1.85-4.35, P<0.001). All these factors have been previously associated with an increased risk of TRA in EBC.
TRA would seem to be particularly relevant to ER+ patients, particularly in the under-50 age group, who would be mostly pre-menopausal at treatment initiation. In the landmark population analysis $(\mathrm{N}=2,844)$ in this study, the TRA cohort was found to have a statistically significant interaction with ER status with regard to DFS $\left(\mathrm{P}_{\text {interaction }}=\right.$ $0.007)$ and $\mathrm{OS}\left(\mathrm{P}_{\text {interaction }}=0.003\right)$. In HER $2+\mathrm{HR}+$ patients $(\mathrm{N}=1,679)$, 6-year DFS rates were $86.6 \%$ vs. $78 \%$ (adjusted HR: 0.58 , 95\% CI: 0.45-0.76), and OS rates were 95.6\% versus $92.2 \%$ (adjusted HR: 0.63, 95\% CI: $0.40-0.99$ ) in the TRA cohort versus the no-TRA cohort, respectively. This difference was not seen in the HER2+ HR- patients $(\mathrm{N}=1,183)$, for either DFS (adjusted HR: 0.92, 95\% CI: 0.701.20 ) or OS (adjusted HR: 1.03, 95\% CI: 0.68-1.56). Notably however, the baseline characteristics differed significantly $(\mathrm{P}<0.001)$ between the distribution of $\mathrm{HR}+(62.7 \%$ vs. $47.7 \%)$ and HR- (37.4\% vs. 52.3\%) patients between the TRA cohort versus the no TRA cohort, respectively.

HER2 protein overexpression has been considered the predominant driver of oncogenesis in HER2+ breast cancers since the early trastuzumab studies, leading to some oncologists questioning the absolute importance of hormone receptor status in this patient population (8). Indeed, current treatment paradigms diverge based on HER2 status, which overcomes the hormonal status in EBC. In fact, even small-sized resected HER2+ tumours should receive upfront chemotherapy (and trastuzumab) regardless of HR status, with subsequent endocrine therapy based on hormone receptor status. By contrast, for HER2 negative (HER2-), HR+ resected tumours of certain dimensions the available recurrence scores (e.g., Oncotype $\mathrm{Dx}^{\circledR}$ ) are both prognostic and predictive, and many women can avoid chemotherapy based on low risk scores (9).

Interestingly, the more recently published findings from the intermediate risk group of the TAILORx study demonstrated some benefit to chemotherapy in women under the age of 50 (10). It is feasible that chemo-induced TRA in this population may account for the findings seen in the updated TAILORx study and this warrants further assessment. This would correlate with the results of the Suppression of Ovarian Function Trial (SOFT) highlighting the benefits of ovarian suppression (11). In SOFT, the 8 -year DFS rate was $78.9 \%$ with tamoxifen alone and $83.2 \%$ with tamoxifen plus ovarian suppression $(\mathrm{P}=0.009)$. The 8 -year OS rate was $91.5 \%$ with tamoxifen alone and $93.3 \%$ with tamoxifen plus ovarian suppression $(\mathrm{P}=0.01)$. Interestingly, the women who remained premenopausal after chemotherapy in SOFT, had lower OS rates at 85.1\% 
and $89.4 \%$, for tamoxifen alone and tamoxifen plus ovarian suppression, respectively. And while both SOFT and TAILORx excluded HER2+ patients, the results support the idea that TRA impacts outcomes.

The impact of chemotherapy on the menstrual cycle and the common side effect of TRA is well described, and the effect of TRA on DFS and OS has also previously been established, for instance, in the NSABP-30 study, where amenorrhoea improved DFS (HR: $0.65, \mathrm{P}<0.001$ ) and OS (HR: $0.72, \mathrm{P}=0.04$ ) regardless of the treatment regimen and hormone receptor status $(12,13)$. In the study by Lambertini and coll., the timing of chemotherapy and $\mathrm{T}$ or $\mathrm{L}$ (sequential versus concurrent) was a significant risk factor for the development of TRA (odds ratio: 1.58, 95\% CI: 1.34-1.86, $\mathrm{P}<0.001$ ), but was not significant in the univariate landmark OS analysis (hazard ratio: 0.82, 95\% CI: $0.62-1.10, \mathrm{P}=0.19$ ). Timing of chemotherapy was therefore omitted from the multivariate analysis on OS. Given the recent findings on the impact of timing of trastuzumab on patients' long-term outcome, with a detrimental effect of the delayed initiation of trastuzumab, it would have been interesting to see that variable assessed in the multivariate analysis (14). There was also a significantly younger cohort of patients in the noTRA cohort (median age: $38 v s .45$ years, $\mathrm{P}<0.001$ ) in this study which their better ovarian reserve likely accounts for.

Given the recent results of the NEOSPHERE and APHINITY trials of pertuzumab plus trastuzumab, along with the ALTTO and NeoALTTO results on lapatinib and trastuzumab and the BETH study investigating the addition of bevacizumab to trastuzumab, the timing of the study by Lambertini $e t a l$. is also relevant given the current debates regarding dual- $v s$ s single-agent HER2-blockade in EBC (4,6,1518). Gonadotoxicity and TRA is an important consideration for these patients and assessments of TRA should be part of further studies in this area. Retrospective analyses on TRA from the datasets from these studies may be forthcoming but future studies with novel anti-HER2 agents and combinations should also include pre-planned analyses on TRA and its impact on outcomes. A quicker and more cost-effective approach might be a combined retrospective analysis from published studies in the EBC setting, including those listed above, the findings from which could prove very insightful.

As yet, despite the efforts of Lambertini and coll., there is no evidence to suggest that HER2-targeted agents impact significantly on TRA and while evidence is gathering that TRA impacts outcomes of patients with HER2+ ER+ EBC, this is not conclusive. In the study by Lambertini and coll. there was a significant difference with regard to the treatment characteristics between the TRA and no-TRA cohorts, particularly with regard to the timing $(\mathrm{P}<0.001)$ and type $(\mathrm{P}<0.001)$ of chemotherapy. As we now know that earlier use of trastuzumab is associated with improved outcomes (14), the significantly higher rates of sequential chemotherapy and HER2-targeted agents in the no-TRA cohort $(60.4 \%$, vs. $43.3 \%$ in the TRA cohort, $\mathrm{P}<0.001$ ) is a considerable confounder. As trastuzumab should be commenced at the earliest possible opportunity as standard of care, the sequential design (non-trastuzumab containing chemotherapy followed by trastuzumab or trastuzumab-containing chemotherapy) is not applicable to the majority of current or future patients and potentially confounded the results seen here with regard to DFS and OS. Again, this would seem to imply that any real benefit seen here is secondary to TRA in itself and not to the different anti-HER2 agents studied.

In summary, while this study by Lambertini et al. does provide some evidence that TRA may matter in the treatment of HER2 + ER+ EBC, the absolute magnitude of this effect, particularly with regard to the timing of chemotherapy and the type of HER2-targeted agents used as well as single versus dual anti-HER2 therapy, needs to be further assessed. This analysis is thought-provoking and may be hypothesis-generating for future studies but is not practice-changing and should not be considered as conclusive. A combined analysis and/or a metanalysis of the impact of TRA on outcomes in the recently published adjuvant HER2-targeted agent trials is called for.

\section{Acknowledgments}

Funding: None.

\section{Footnote}

Provenance and Peer Review: This article was commissioned by the editorial office, Translational Cancer Research. The article did not undergo external peer review.

Conflicts of Interest: GG has the following disclosuresTravel funding: Roche, Pfizer, MSD; Speaker honoraria: Roche, Genomic Health, Novartis; Advisory roles: Roche, Amgen, BMS. JG has the following disclosures-Travel funding: Astellas, Pfizer, AbbVie; Speaker honoraria: Novartis, BMS; Advisory roles: Roche, BMS.

Ethical Statement: The authors are accountable for all aspects of the work in ensuring that questions related 
to the accuracy or integrity of any part of the work are appropriately investigated and resolved.

Open Access Statement: This is an Open Access article distributed in accordance with the Creative Commons Attribution-NonCommercial-NoDerivs 4.0 International License (CC BY-NC-ND 4.0), which permits the noncommercial replication and distribution of the article with the strict proviso that no changes or edits are made and the original work is properly cited (including links to both the formal publication through the relevant DOI and the license). See: https://creativecommons.org/licenses/by-nc-nd/4.0/.

\section{References}

1. Slamon DJ, Eiermann W, Robert NJ, et al. Abstract S5-04: Ten year follow-up of BCIRG-006 comparing doxorubicin plus cyclophosphamide followed by docetaxel $(\mathrm{AC} \rightarrow \mathrm{T})$ with doxorubicin plus cyclophosphamide followed by docetaxel and trastuzumab $(\mathrm{AC} \rightarrow \mathrm{TH})$ with docetaxel, carboplatin and trastuzumab (TCH) in HER2 + early breast cancer. Cancer Res 2016;76:S5-04-S5-04.

2. Perez EA, Romond EH, Suman VJ, et al. Four-Year FollowUp of Trastuzumab Plus Adjuvant Chemotherapy for Operable Human Epidermal Growth Factor Receptor 2Positive Breast Cancer: Joint Analysis of Data From NCCTG N9831 and NSABPB-31. J Clin Oncol 2011;29:3366-73.

3. Cameron D, Piccart-Gebhart MJ, Gelber RD, et al. 11 years' follow-up of trastuzumab after adjuvant chemotherapy in HER2-positive early breast cancer: final analysis of the HERceptin Adjuvant (HERA) trial. Lancet 2017;389:1195-205.

4. von Minckwitz G, Procter M, de Azambuja E, et al. Adjuvant Pertuzumab and Trastuzumab in Early HER2Positive Breast Cancer. N Engl J Med 2017;377:122-31.

5. Slamon D, Eiermann W, Robert N, et al. Adjuvant Trastuzumab in HER2-Positive Breast Cancer. N Engl J Med 2011;365:1273-83.

6. Piccart-Gebhart M, Holmes E, Baselga J, et al. Adjuvant Lapatinib and Trastuzumab for Early Human Epidermal Growth Factor Receptor 2-Positive Breast Cancer: Results From the Randomized Phase III Adjuvant Lapatinib and/ or Trastuzumab Treatment Optimization Trial. J Clin Oncol 2016;34:1034-42.

7. Lambertini M, Campbell C, Bines J, et al. Adjuvant AntiHER2 Therapy, Treatment-Related Amenorrhea, and Survival in Premenopausal HER2-Positive Early Breast Cancer Patients. J Natl Cancer Inst 2018. [Epub ahead of print].
8. Slamon DJ, Leyland-Jones B, Shak S, et al. Use of Chemotherapy plus a Monoclonal Antibody against HER2 for Metastatic Breast Cancer That Overexpresses HER2. N Engl J Med 2001;344:783-92.

9. Sparano JA, Gray RJ, Makower DF, et al. Prospective Validation of a 21-Gene Expression Assay in Breast Cancer. N Engl J Med 2015;373:2005-14.

10. Sparano JA, Gray RJ, Makower DF, et al. Adjuvant Chemotherapy Guided by a 21-Gene Expression Assay in Breast Cancer. N Engl J Med 2018;379:111-21.

11. Francis PA, Regan MM, Fleming GF, et al. Adjuvant ovarian suppression in premenopausal breast cancer. $\mathrm{N}$ Engl J Med 2015;372:436-46.

12. Swain SM, Jeong JH, Wolmark N. Amenorrhea from breast cancer therapy--not a matter of dose. N Engl J Med 2010;363:2268-70.

13. Swain SM, Jeong JH, Geyer CE Jr, et al. Longer therapy, iatrogenic amenorrhea, and survival in early breast cancer. N Engl J Med 2010;362:2053-65.

14. Gullo G, Walsh N, Fennelly D, et al. Impact of timing of trastuzumab initiation on long-term outcome of patients with early-stage HER2-positive breast cancer: the "one thousand HER2 patients" project. Br J Cancer 2018;119:374-80.

15. Gianni L, Pienkowski T, Im YH, et al. 5-year analysis of neoadjuvant pertuzumab and trastuzumab in patients with locally advanced, inflammatory, or early-stage HER2-positive breast cancer (NeoSphere): a multicentre, open-label, phase 2 randomised trial. Lancet Oncol 2016;17:791-800.

16. de Azambuja E, Holmes AP, Piccart-Gebhart M, et al. Lapatinib with trastuzumab for HER2-positive early breast cancer (NeoALTTO): survival outcomes of a randomised, open-label, multicentre, phase 3 trial and their association with pathological complete response. Lancet Oncol 2014;15:1137-46.

17. Gleeson JP, Keegan NM, Morris PG. Adding Pertuzumab to Trastuzumab and Taxanes in HER2 positive breast cancer. Expert Opin Biol Ther 2018;18:251-62.

18. Slamon DJ, Swain SM, Buyse M, et al. Abstract S1-03: Primary results from BETH, a phase 3 controlled study of adjuvant chemotherapy and trastuzumab \pm bevacizumab in patients with HER2-positive, node-positive or high risk node-negative breast cancer. AACR; 2013.

Cite this article as: Gullo G, Gleeson JP. Treatment-induced amenorrhoea for patients with HER2-positive early breast cancer: does it matter? Transl Cancer Res 2018;7(6):1357-1360. doi: $10.21037 /$ tcr.2018.09.06 